\title{
Valoración del Desempeño de un Dispositivo de Autoalimentación Diseñado para la Evaluación Operativa de Electrodos Revestidos
}

\author{
(Self-Feed Device Behaviour Valuation Designed for Assessment of Operability of Covered Electrodes)
}

\author{
Alejandro García Rodriguez ${ }^{1}$, Carlos René Gómez Pérez ${ }^{1}$, Jorge Víctor Miguel Oria ${ }^{1}$, Oscar Miguel Rivera Borroto ${ }^{2}$, Angel Sánchez \\ Roca $^{3}$ \\ ${ }^{1}$ Universidad Central "Marta Abreu” de las Villas, Centro de Investigaciones de Soldadura (CIS) /Ministerio de Educación \\ Superior(MES),SantaClara,VillaClara,Cuba alejo2@uclv.edu.cu,crene@uclv.edu.cu, jorgev@uclv.edu.cu, \\ sanchez@rect.uo.edu.cu \\ ${ }^{2}$ Universidad Central "Marta Abreu" de las Villas, Centro de Estudios Informáticos (CEI)/Ministerio de Educación Superior (MES), \\ Santa Clara, Villa Clara,Cuba OscarRb@uclv.edu.cu \\ ${ }^{3}$ Universidad de Oriente. Departamento de Tecnología/ Ministerio de Educación Superior (MES), Santiago de Cuba, Cuba.
}

\section{Resumen}

El presente trabajo tiene como objetivo valorar la efectividad de un dispositivo de autoalimentación para electrodos revestidos. El mismo es capaz de eliminar perturbaciones inherentes a operarios o sistemas de control automático, para el estudio del comportamiento de electrodos revestidos. La conjugación de diferentes métodos, tales como el análisis estadístico del tiempo de duración del cortocircuito, el análisis metalográfico (penetración, zona afectada por el calor, coeficiente de forma y distancia promedio entrefrentes de solidificación del cordón), junto al análisis del comportamiento de los parámetros eléctricos primarios del arco y los índices de consumo del electrodo, permiten obtener relaciones entre el comportamiento eléctrico del arco, el proceso de transferencia de masa y la apariencia del cordón. Esto permite extraer criterios sobre la estabilidad del proceso y el desempeño del dispositivo de alimentación empleado. Se obtiene una metodología estadística, apropiada para el procesamiento del parámetro "duración del cortocircuito", a partir de un adecuado ajuste de las distribuciones empiricas a un modelo Lognormal. El dispositivo de autoalimentación para electrodos revestidos, permite la ejecución repetible y reproducible de cordones de soldadura en posición plana, manteniendo la longitud del arco en función de las características físico-químicas del consumible evaluado en regímenes de trabajo de 125A, 140A y 160A.

Palabras clave: Electrodos revestidos, operatividad, dispositivo autoalimentación, procesamiento estadístico, parámetros eléctricos.

\begin{abstract}
The objective of the present work is to evaluate the effectiveness of a self-feed device for covered electrodes. This device is able of eliminate disturbances from manual operation or the use of automatic control systems during the study of covered electrode behavior. The conjugation of different methods like short-circuit time statistical analysis, metallographic analysis (penetration, heat affected zone, shape and mean distance between successive solidification fronts) together with the analysis of the electric arc and electrode melting parameters allow us to obtain relationships between arc behavior, mass transfer processes and bead appearance. This permits the extraction of criteria to describe process stability and the behavior of the feed device employed. An appropriate statistical methodology was obtained to process the parameter "duration of short-circuit" through an appropriate adjustment of empirical distributions to a lognormal model. The self-feed device for covered electrode welding allows the repeatable and reproducible execution of welding beads in the flat position, keeping the arc length based on physical and chemistry characteristics of the electrode during welding trials using $125 \mathrm{~A}, 140 \mathrm{~A}$, and $160 \mathrm{~A}$.
\end{abstract}

Key-words: Covered electrodes, operability, self-feed device, statistical processing, electrics parameters.

\section{Introducción}

Uno de los problemas en la valoración de la operatividad eléctrica de los electrodos revestidos, proviene del hecho de que la corriente de soldadura dependa de la longitud de arco, entre otros factores. Cuando se establece un arco con un electrodo revestido determinado, es necesario alimentarlo a medida que se consume, tratando de mantener constante la longitud de arco.

(Recebido em 06/02/2008; Texto Final em 22/01/2009).
Si bien lograr esto es dificultoso por parte del operario en la soldadura manual, esta premisa de la soldadura resulta también difícil de lograr automáticamente, ya que la longitud de arco es muestreada indirectamente a partir del voltaje o la corriente de soldadura, y los lazos y dispositivos de control poseen una cierta inercia [1].

Ante la necesidad de implementar un sistema para la evaluación de la operatividad eléctrica específica del electrodo revestido, ha sido preciso desarrollar un estudio sobre el comportamiento de los parámetros eléctricos del arco durante la soldadura SMAW (Shielded Metal Arc Welding). Algunos 
sistemas y métodos [2-5] de estudio de la estabilidad del arco eléctrico con electrodo revestido en el modo de transferencia metálica por cortocircuitos, han sido reportados en artículos de revistas científicas y libros. El uso indiscriminado de estos métodos, puede conducir a distorsiones en los resultados de la caracterización [6], haciéndose interesante retomar el tema de la evaluación de la operatividad eléctrica de los electrodos revestidos.

El presente trabajo tiene como objetivo valorar la efectividad de un sistema de autoalimentación diseñado, que elimina perturbaciones inherentes a operarios o sistemas de control automático, para el estudio del comportamiento de la operatividad en electrodos revestidos.

\section{Materiales y Métodos}

\subsection{Dispositivo de alimentación por gravedad}

Dispositivos de alimentación por gravedad han sido utilizados para la soldadura con electrodos de carbón [7] y para la soldadura con electrodo revestido en aplicaciones de la industria naval $[8,9]$, así como para el estudio de propiedades de la soldadura subacuática mojada $[10,11]$. No obstante, la eficacia de este dispositivo en la obtención de las características operativas intrínsecas del electrodo no ha sido suficientemente estudiada.

Bajo este principio, en el Centro de Investigaciones de Soldadura, de la Universidad Central "Marta Abreu"de Las Villas, se ha construido un dispositivo similar (Figura 1), para la investigación. El dispositivo consta de una base metálica (3), que sostiene un soporte vertical (2) por el que se desliza por gravedad la corredera (6) solidaria al brazo (9), que soporta la tenaza (8), sosteniendo al electrodo (5) por el extremo opuesto al cordón de soldadura (11) y, a la vez, suministrando el contacto eléctrico con el conductor (10), proveniente de la fuente de soldadura, mediante una unión roscada desarmable (7). La tenaza (8) gira respecto a un eje pasante perpendicular a la pieza (9), lo que permite ajustar el ángulo de incidencia $(\alpha)$ del electrodo respecto a la placa a soldar (4). La varilla (1) limita el movimiento radial del electrodo restringiendo las oscilaciones en la trayectoria del cordón (11).

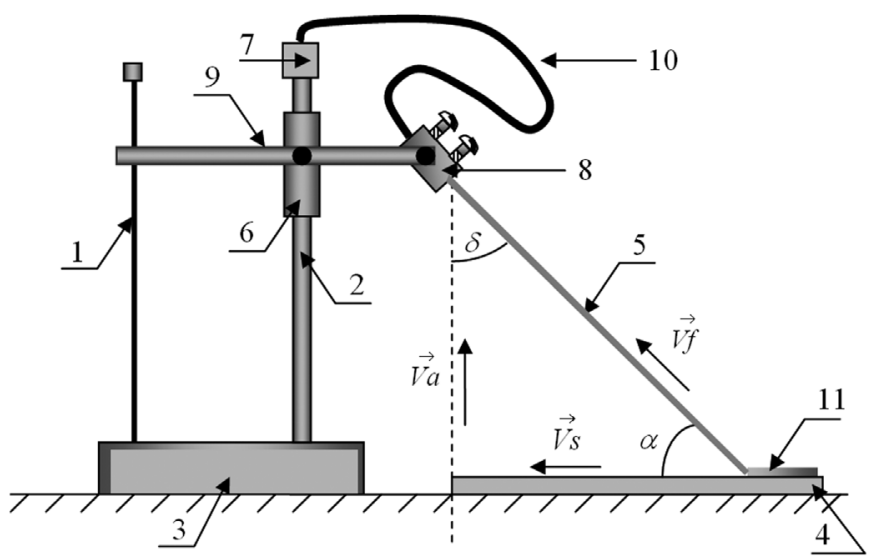

Figura 1. Esquema del dispositivo de autoalimentación por gravedad.
$\mathrm{Al}$ iniciarse el arco de soldadura, el consumo del electrodo hace que la corredera (6) baje gravitacionalmente, mientras la punta del electrodo se consume por el intenso calor del arco.

En la Figura 1, se representa un triángulo vectorial de velocidades dónde: $\overrightarrow{V f}$, es la velocidad de fusión del electrodo o la velocidad de alimentación del electrodo.; $\overrightarrow{V_{S}}$, es la velocidad de soldadura; $\overrightarrow{V a}$, es la velocidad con que baja la tenaza (8) Teniendo en cuenta el teorema de Pitágoras y las respectivas consideraciones trigonométricas, en un análisis vectorial del polígono de velocidades formado, pueden relacionarse las velocidades (Ecuación 1 y 2).

$$
\begin{aligned}
& V f=\sqrt{V a^{2}+V s^{2}} \\
& \tan (\alpha)=\left(\frac{V a}{V s}\right)
\end{aligned}
$$

Ecuación 1

Ecuación 2

El empleo de estas ecuaciones permitiría, en caso de necesitarse monitorear la velocidad de soldadura $\left(\overrightarrow{V_{S}}\right)$, medir la velocidad $(\overrightarrow{V a})$ con que el brazo (9) baja, lo cual puede hacerse acoplando un dispositivo óptico al soporte. También es posible estimar un promedio de la velocidad de soldadura midiendo el tiempo total de la soldadura (ts) y la longitud del cordón (1), considerando el proceso de soldadura como un movimiento rectilíneo y uniforme.

$V s=\frac{l}{t s}$

Ecuación 3

La velocidad de soldadura $(\overrightarrow{V S})$ puede variarse según el ángulo de incidencia $(\alpha)$ del electrodo y puede calcularse según la Ecuación 3. Para un mismo electrodo la eficiencia del proceso está relacionada al ángulo de incidencia del electrodo $(\alpha)$ respecto a la placa a soldar, la longitud de arco y el aporte térmico.

Durante el empleo del dispositivo (Figura 1), el peso de algunos de sus componentes recae sobre el borde de la copa de revestimiento, por lo que existe un compromiso entre su peso, las características termo físicas del material del revestimiento y su espesor. La corredera (6), el brazo (9) y la pinza (8) deben ser construidos de materiales ligeros para evitar esfuerzos innecesarios sobre el revestimiento que puedan quebrarlo.

Dentro de los parámetros de calidad del electrodo revestido se encuentra la excentricidad del revestimiento, así como la homogeneidad de su composición química y mineralógica, entre otros.

Cuando se utiliza el dispositivo de alimentación por gravedad en la soldadura con electrodo revestido, la longitud de arco queda determinada por el ángulo de incidencia $(\alpha)$ y la profundidad de la copa en la punta del electrodo. La profundidad de la copa depende de las condiciones energéticas del proceso y de las características termo físicas del recubrimiento del electrodo, fundamentalmente.

Cualquier anomalía que se presente en el recubrimiento, entiéndase: excentricidad, poros, falta de homogeneidad, etc.; debe reflejarse inequívocamente en variaciones en los parámetros, sin la presencia de perturbaciones producidas por algún tipo de alimentación forzada del electrodo. Por tanto, el 
equipo de alimentación por gravedad empleado para la evaluación operativa de electrodos revestidos, a través del sensado y el procesamiento del voltaje y la corriente del arco eléctrico de soldadura, puede conducir a la obtención de mediciones de los parámetros del proceso en dependencia de las características termo físicas del consumible, que se apoya en el borde de su revestimiento durante la operación.

\subsection{Planeación Experimental}

Para comprobar la eficacia del sistema de alimentación propuesto y demostrar que los resultados obtenidos de la soldadura con electrodos comerciales son consistentes se seleccionaron, a partir de un lote de electrodos de la clasificación E6013 [12] del fabricante RUTH (España), 9 muestras aleatorias sin reposición de electrodos (Ø $4 \mathrm{~mm}$ ), certificados estos para su uso en el rango de corriente desde $120 \mathrm{~A}$ a $160 \mathrm{~A}$. Se opta por una planificación experimental correspondiente a un diseño factorial de 3 niveles, con dos réplicas independientes. Los diferentes regímenes de corriente (Tabla 1), conforman los 3 niveles de la variable o factor independiente "Corriente". La literatura especializada [2-5] reconoce el "tiempo de duración del cortocircuito", como un parámetro importante en la caracterización de los fenómenos de transferencia metálica, obtenido a partir del procesamiento del voltaje y la corriente de soldadura caracterizando en parte el fenómeno de transferencia metálica.

Tabla 1. Valores de corriente a los que se someten los electrodos en cada uno de los tres ensayos.

\begin{tabular}{|c|c|c|c|}
\hline Experimento & 1 & 2 & 3 \\
\hline Corriente de Soldadura (A) & 125 & 140 & 160 \\
\hline
\end{tabular}

La fuente de corriente alterna impone reiteradas inversiones de polaridad en el arco a un ritmo de 120 veces por segundo. Este aspecto hace que se encienda y apague el arco eléctrico en sucesivas oportunidades, pudiéndose evaluar el comportamiento eléctrico del consumible en condiciones de inestabilidad sostenidas.

\subsection{Instalación Experimental}

La instalación experimental (Figura 2), está compuesta por un acondicionador de señales (1), que recibe entre sus terminales (e) y (f), la señal de voltaje del transformador de soldadura (3) marca AIRCO 220V AC.

La corriente de soldadura es regulable mediante la variación del área efectiva del núcleo del transformador; presentando un voltaje de 80 Volt en vacío. La señal de corriente es tomada de los extremos del sensor de corriente tipo "Shunt" (4) de $60 \mathrm{mV}$ a $250 \mathrm{~A}$, clase 0,5 ; acoplándola a los terminales (c) y (d) del acondicionador (1). La mesa de trabajo (7), está conectada al terminal de tierra $(\mathrm{G})$ de la fuente de soldadura, a través del sensor de corriente. El terminal (B) de la fuente de soldadura está conectado al porta electrodo del dispositivo de autoalimentación (8) y la placa (6), sobre la que se efectúa el depósito, está debidamente conectada eléctricamente a la mesa de trabajo (7).

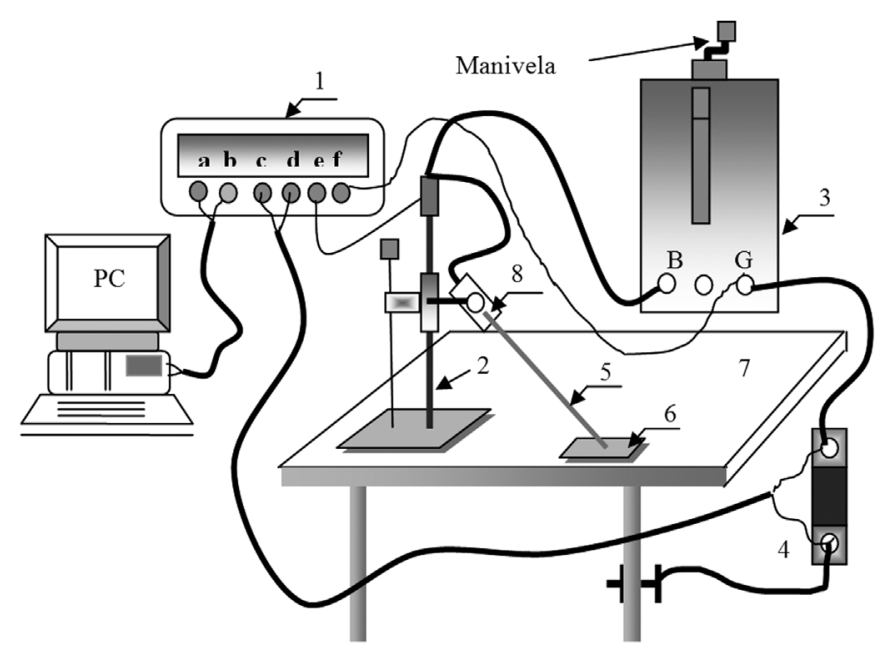

Figura 2. Representación esquemática de la instalación experimental.

El acondicionador de señales (1) brinda en sus terminales (a) y (b), las señales de voltaje y corriente, preparadas para su entrada en la tarjeta de adquisición de datos, acoplada internamente a la computadora.

El sistema de adquisición de datos Advantech PCLD8710 [13] está compuesto por una bornera para la conexión de terminales (PCLD-8710) y una tarjeta de adquisición de datos (PCI-1710).

La bornera de grado industrial PCLD-8710, con bornes atornillados, permite un fácil montaje de las tarjetas PCI-1710 o PCI-1710HG y conexión SCSI-II de 68 pines, que incluyen circuitos de compensación de junta fría para la medición directa con termopares, y espacios reservados para circuitos acondicionadores de señales, tales como: filtros paso bajo, atenuadores de tensión y de corriente [14].

Por su parte, la tarjeta de adquisición de datos PCI-1710 posee transferencia de datos mediante el BUS-PCI, una frecuencia de muestreo de hasta $100 \mathrm{kHz}$, un convertidor A/D de 12 bits, 16 canales de entrada digital, 16 canales de salida digital, contador y temporizador programable y 2 canales de salida analógica.

Entre las especificaciones de las entradas analógicas se cuenta con las siguientes: 16 canales de entrada simple u 8 entradas diferenciales o combinaciones; una frecuencia de muestreo de 100 mil muestras por segundo; tiempo de conversión 8 microsegundos; tensión máxima de entrada, $\pm 15 \mathrm{~V}$; impedancia de entrada, 1 millón de Ohms por cada 5 pico Faradios [15].

\subsection{Proceder Experimental}

Las corridas experimentales fueron realizadas en la instalación (Figura 2) para el sensado y procesamiento de las señales eléctricas del arco de soldadura. Se realizaron soldaduras con corriente alterna sobre placas de 200x50x10 mm de acero al carbono CT-3, según las normas Rusas y Ucranianas "Gostandar". El dispositivo de alimentación y las placas a soldar fueron fijados mediante tornillos a la mesa de trabajo empleándose la siguiente técnica operatoria:

1. Comprobación de conexión eléctrica entre: la placa a soldar (6) 
y la mesa de trabajo (7); el terminal aterrado de la fuente $(\mathrm{G})$ y la mesa de trabajo (7) y el terminal no aterrado de la fuente (V) a la tenaza (8). Además, se debe fijar adecuadamente el electrodo (5) al soporte (8), y mediante el tornillo de ajuste (12), fijar el ángulo $(\alpha)$ de incidencia del electrodo en $60^{\circ}$, para los experimentos.

2. La corriente alterna de la fuente es ajustada manipulando la manivela de control de corriente del transformador de soldadura, observando en la computadora su valor rms.

3. Se inicia el arco y se ejecuta el depósito completo, mientras se adquieren y registran los valores digitales de voltaje y corriente del arco.

4. La escoria y la placa son relacionadas junto a la colilla con un identificador. Se observa el grado de facilidad de desprendimiento de la escoria.

5. Los cordones se realizan sobre placas frías $\left(\approx 30{ }^{\circ} \mathrm{C}\right)$ utilizando electrodos E6013, de la marca RUTH, de $4 \mathrm{~mm}$.

6. Se determinan los parámetros de consumo del electrodo [16].

\section{5. Procesamiento Digital de las Señales}

Las señales se procesan con el paquete de programas MATLAB 7.0, utilizando las Herramientas de Procesamiento Digital de Señales (PDS) y la implementación de un programa para la detección automática de la frecuencia de cortocircuito y la duración de los mismos, parámetros que caracterizan el comportamiento de la transferencia metálica. Para la detección de la duración de cada cortocircuito se requiere el análisis en el histograma de frecuencias del voltaje de soldadura para localizar el valor límite por debajo del cuál se va a considerar que existe un cortocircuito. En este caso se determina que el voltaje umbral puede ser tomado como $\pm 5 \mathrm{~V}$, por lo que el algoritmo debe determinar el tiempo en que la señal de voltaje permanece por debajo de este valor para cada cortocircuito; además, debe anotar el momento en que ocurre la entrada para el cálculo posterior de la frecuencia de cortocircuito, una vez calculado el valor promedio del período de ocurrencia del corto.

El programa fue realizado en el código disponible en MATLAB 7.0, compilado y probado en el mismo paquete de programas, obteniéndose los resultados y comprobándose, mediante la revisión del comportamiento de la señal en el tiempo.

\subsection{Procesamiento Estadístico del Parámetro "Duración del Cortocircuito"}

Este parámetro se relaciona con la cantidad de metal aportado por unidad de tiempo y, por tanto, con la productividad del proceso.

Para el procesamiento estadístico de los datos de duración del cortocircuito (Tabla 2), obtenidos en los ensayos descritos, se ha utilizado el Software STATGRAPHICS Centurion XV, versión 15.1.02.

Tabla 2. Resultado de las pruebas de normalidad, cálculo de estadígrafos y pruebas de ajuste de las distribuciones empíricas

\begin{tabular}{|c|c|c|c|c|c|c|c|}
\hline \multirow{2}{*}{ Ensayo } & \multicolumn{2}{|c|}{$\begin{array}{c}\text { Pruebas de normalidad } \\
\text { Shapiro-Wilk W }\end{array}$} & \multicolumn{3}{|c|}{ LogNormal } & \multicolumn{2}{c|}{$\begin{array}{c}\text { Pruebas Kolmogorov-Smirnov } \\
\text { (Lognormal ) }\end{array}$} \\
\hline & Statistic & P-Value & Media(ms) & Std-Dev(ms) & P-Value & No & $\mathrm{Si}$ \\
\hline E160A22 & 0,72 & 0 & 5,71 & 3,38 & 0,0451256 & $\mathrm{x}$ & \\
\hline E160A23 & 0,64 & 0 & 5,24 & 2,8 & 0,222115 & & $\mathrm{x}$ \\
\hline E160A33 & 0,83 & 0 & 5,38 & 2,87 & 0,610693 & & $\mathrm{x}$ \\
\hline E140A16 & 0,66 & 0 & 7,32 & 5,73 & 0,0898782 & & $\mathrm{x}$ \\
\hline E140A15 & 0,74 & 0 & 7,47 & 5,77 & 0,05011 & & $\mathrm{x}$ \\
\hline E140A14 & 0,55 & 0 & 7,65 & 6,14 & 0,415002 & & $\mathrm{x}$ \\
\hline E125A20 & 0,77 & 0 & 9,86 & 8,43 & 0,657347 & & $\mathrm{x}$ \\
\hline E125A19 & 0,9 & 0 & 10,08 & 7,24 & 0,415002 & & $\mathrm{x}$ \\
\hline E125A18 & 0,76 & 0 & 9,89 & 8,49 & 0,716605 & & $\mathrm{x}$ \\
\hline
\end{tabular}

Se emplean criterios del proceder de un análisis de ANOVA estándar, examinándose la normalidad de los datos obtenidos. Así, según el método Shapiro-Wilk W [17] para los nueve ensayos, se observa (Tabla 2) que en ninguno de los casos el valor de probabilidad excede el valor 0,05 , por lo que ninguna de las distribuciones en cuestión puede ser modelada por una distribución normal con un nivel de confianza del $95 \%$.

Sin embargo, las pruebas realizadas según el método de Kolmogorov-Smirnov [18], para determinar si las distribuciones empíricas pueden ser modeladas mediante una distribución Lognormal, arrojan que en ocho de las distribuciones el valor de probabilidad asociado (P-value) excede el valor de 0,05 , por lo que sí es posible modelar estas distribuciones usando una aproximación Lognormal.

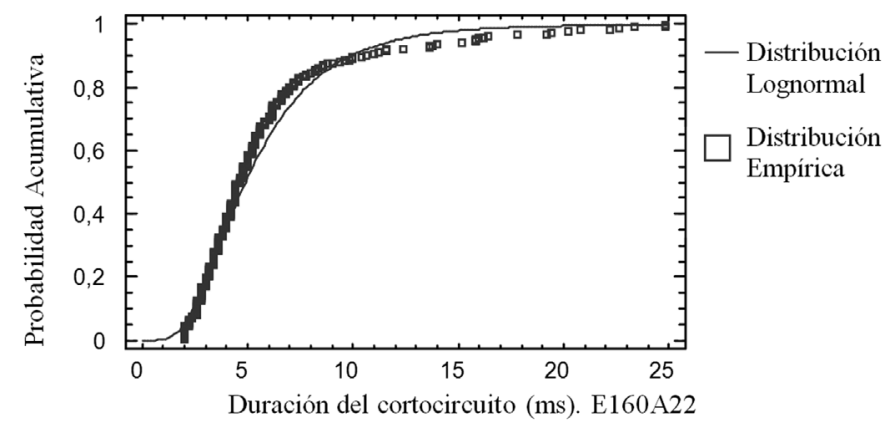

Figura 3. Función de densidad acumulativa para el parámetro duración del cortocircuito para el ensayo E160A22. 
En el caso de la muestra E160A22 puede apreciarse gráficamente (Figura 3) que, en efecto, una pequeña perturbación no aleatoria en las condiciones fenomenológicas pudo incidir en los resultados anteriores, sin embargo, es evidente la similitud (cercanía) entre la distribución empírica y la Lognormal.

De forma implícita en el análisis anterior, el procedimiento de cálculo se realiza partiendo de la transformación $Y=\ln [X]$ en cada uno de los datos muestrales. El resultado de esta transformación resultó en subconjuntos numéricos con distribuciones normales, por tanto, es posible calcular la media $(\bar{y})$ y la desviación estándar $\left(S_{Y}\right)$, según las Ecuaciones 6 y 7, respectivamente.

$\bar{y}=\sum_{i=1}^{n} \frac{\ln \left[x_{i}\right]}{n}$

Ecuación 6

$S_{Y}=\sqrt{\frac{\sum_{i=1}^{n}\left(\ln \left(x_{i}\right)-\bar{x}\right)^{2}}{(n-1)}}$

Ecuación 7

Si se estima $\mu_{Y}=\bar{y}$ y $\sigma_{Y}=s_{Y}$ entonces, para calcular la media $\left(\mu_{X}\right)$ y la desviación estándar $\left(\sigma_{X}\right)$ de la variable aleatoria original $X$ se pueden deducir las Ecuaciones 8 y 9 [19], correspondientemente.

$\mu_{X}=e^{\left(\mu_{Y}+\frac{\sigma_{Y}^{2}}{2}\right)}=e^{\left(\bar{y}+\frac{s_{Y}^{2}}{2}\right)}$

Ecuación 8

$\sigma_{X}=\sqrt{e^{\left(2 \mu_{Y}+2 \sigma_{Y}^{2}\right)}-e^{\left(2 \mu_{Y}+\sigma_{Y}^{2}\right)}}=\sqrt{e^{\left(2-\bar{y}+2 s_{Y}^{2}\right)}-e^{\left(2-\bar{y}+s_{Y}^{2}\right)}}$

Ecuación 9

La posibilidad de apoyarse en una transformación logarítmica, cuyo resultado es modelable por la conocida función gaussiana, coincide con una distribución Lognormal, que permite corroborar los estadígrafos descriptivos (Tabla 2) en cada uno de los 9 subconjuntos de datos obtenidos, apoyados en el Toolbox Statistic de MATLAB 7.0.

El hecho de modelar cada subconjunto o muestra de datos mediante la distribución Lognormal, a través de los distintos niveles de corriente, sugiere la concurrencia de los mismos mecanismos fenomenológicos del proceso y sus relaciones internas independientemente de la influencia cuantitativa de estos niveles de corriente (Figuras 4 y 5).

En la medida en que se incrementa el valor de la corriente, los valores de la desviación estándar disminuyen (Tabla 2), lo que puede asociarse al hecho de que en el experimento con 160 A se alcanza el régimen de transferencia metálica más estable, lo que se corrobora durante la ejecución del experimento y el análisis metalográfico del cordón (Tabla 3 ).

La función de distribución de densidad se aprecia muy bien definida (Figura 4) en los grupos de corriente de soldadura (160 A, 140 A y 125 A).

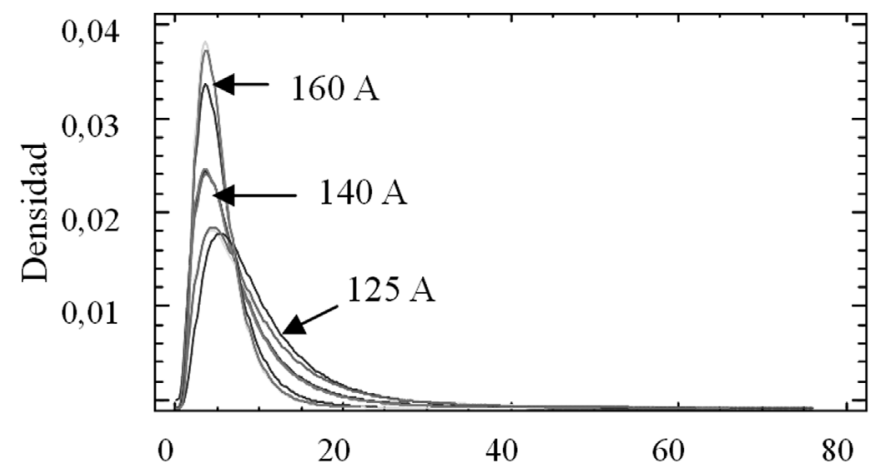

Tiempo de Duración del Cortocircuito (ms)

Figura 4. Densidad probabilística del parámetro, "Duración del Cortocircuito" para los nueve ensayos realizados en tres niveles de corriente de soldadura.

La aproximación de las distribuciones de densidad (Figura 4) y las distribuciones acumulativas (Figura 5) en las réplicas independientes de cada punto experimental sugieren un adecuado nivel de repetibilidad y reproducibilidad en el procedimiento.

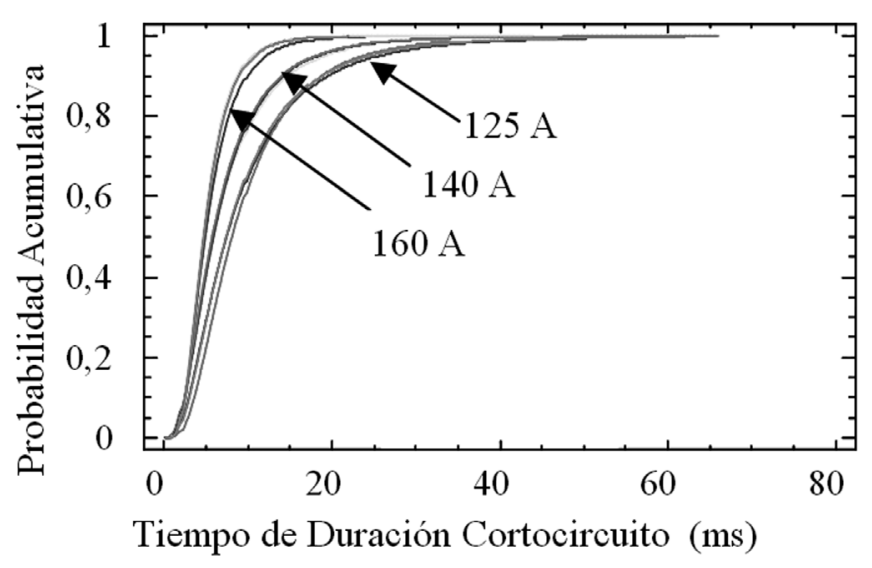

Figura 5. Funciones de Probabilidad Acumulativa para el parámetro "Duración del cortocircuito". Ensayos en tres niveles de corriente de soldadura.

\subsection{Análisis metalográfico}

La dispersión de la duración del cortocircuito en el caso en que se utiliza una corriente de $160 \mathrm{~A}$ es la menor (Figura 4), lo cual indica que el proceso de transferencia de masa es más estable, lo cual debe producir un cordón más uniforme en cuanto a su coeficiente de forma y es constatado por inspección visual de los cordones, lo que define este punto experimental como el punto operativo más eficiente del electrodo. Para estimar el comportamiento de la zona de influencia térmica y la penetración, el ancho y la altura del cordón, se realizaron 10 medidas equidistantes a lo largo de la zona central de los tres cordones obtenidos a 160 A. Se utilizó un microscopio óptico NEOPHOT-32 con una precisión de 0,02 mm (Tabla 3). 
Tabla 3. Valores Promedios y desviaciones estándar (Dev) de la zona afectada térmicamente (ZAT), la penetración (Pen), el ancho (Anch) y el refuerzo promedio (Ref) de los tres cordones logrados en los depósitos a $160 \mathrm{~A}$

\begin{tabular}{|l|l|l|l|l|l|l|l|}
\hline ZIT $(\mathrm{mm})$ & $\operatorname{Dev}(\mathrm{mm})$ & $\operatorname{Pen}(\mathrm{mm})$ & $\operatorname{Dev}(\mathrm{mm})$ & $\operatorname{Anch}(\mathrm{mm})$ & $\operatorname{Dev}(\mathrm{mm})$ & $\operatorname{Ref}(\mathrm{mm})$ & $\operatorname{Dev}(\mathrm{mm})$ \\
\hline 3.69 & 0.17 & 1.11 & 0.12 & 10,26 & 0,35 & 3,00 & 0,14 \\
\hline
\end{tabular}

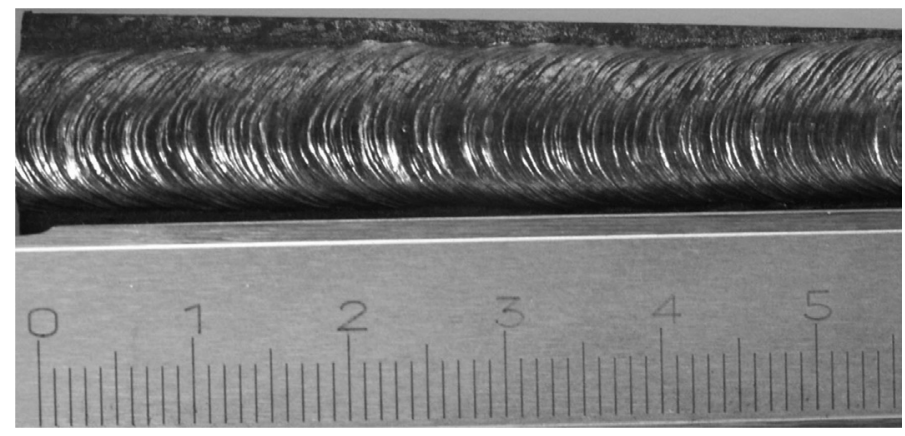

Figura 6. Fotografía de 5 centímetros de la zona central del cordón del experimento E160A23.

Las desviaciones estándar de las mediciones de la ZAT, el ancho y el refuerzo promedio, se producen en menos de un $5 \%$ del valor promedio. La desviación estándar de la penetración se aproxima a un $10 \%$. Los cordones (Figura 6) logrados con el dispositivo poseen apariencias muy superiores a los logrados por un operador en la soldadura manual.

\subsubsection{Medición de la distancia entre los frentes de solidificación}

El traslado de la fuente de calor a través de la plancha, produce frentes de solidificación (Figura 7), (conchas) del metal fundido en la poza de soldadura.

La medición se realizó con un microscopio óptico NEOPHOT$32 \mathrm{a}$ un aumento de $50 \mathrm{X}$, y con una precisión es de $0,02 \mathrm{~mm}$. Se realizaron 40 mediciones de distancias entre conchas de cada uno de los tres cordones obtenidos a $160 \mathrm{~A}$, en la zona central del cordón, brindándose el promedio y desviación estándar en la Tabla 4.

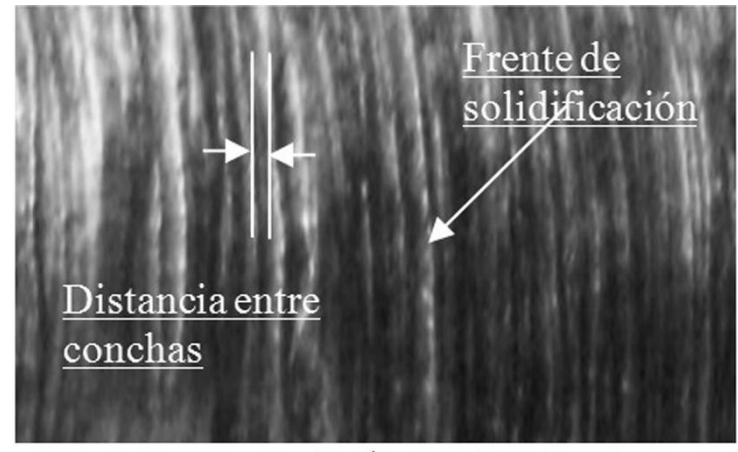

A

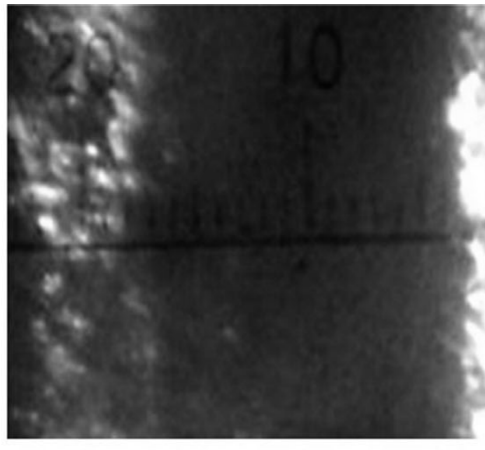

B

Figura 7. Fotografía de un fragmento de la sección central del cordón de soldadura, A-Frentes de solidificación y distancia entre conchas. B-Ampliación de una sección del mismo cordón a un aumento 50X, con una precisión de $0,02 \mathrm{~mm}, 10$ divisiones de la escala en color negro equivalen $0,2 \mathrm{~mm}$.

Tabla 4. Distancia promedio entre conchas y su desviación estándar

\begin{tabular}{|c|c|c|}
\hline \multirow{2}{*}{ Distancia promedio entre conchas } & Promedio $(\mathrm{mm})$ & Desviación Estándar $(\mathrm{mm})$ \\
\cline { 2 - 3 } & 0,12 & 0,07 \\
\hline
\end{tabular}

Tabla 5. Índices de consumo de los electrodos evaluados con $160 \mathrm{~A}$

\begin{tabular}{|c|r|r|r|r|r|r|r|}
\hline & $\alpha f(\mathrm{~g} / \mathrm{A}-\mathrm{h})$ & $\alpha d(\mathrm{~g} / \mathrm{A}-\mathrm{h})$ & $\mathrm{P}(\mathrm{Kg} / \mathrm{h})$ & $\psi(\%)$ & \multicolumn{1}{c|}{$\mathrm{E} \mathrm{( \% )}$} & \multicolumn{1}{c|}{$\mathrm{ED}(\%)$} & \multicolumn{1}{c|}{$\mathrm{Et}(\%)$} \\
\hline 1 & 9,11 & 8,19 & 1,35 & 10,08 & 89,92 & 66,29 & 51,67 \\
\hline 2 & 9,28 & 8,14 & 1,32 & 12,36 & 87,64 & 64,67 & 50,90 \\
\hline 3 & 9,28 & 8,23 & 1,32 & 11,28 & 88,72 & 63,87 & 50,89 \\
\hline $\mathrm{M}$ & 9,22 & 8,19 & 1,33 & 11,24 & 88,76 & 64,94 & 51,15 \\
\hline $\mathrm{D}$ & 0,1 & 0,05 & 0,02 & 1,14 & 1,14 & 1,23 & 0,45 \\
\hline
\end{tabular}

1.- E160A22; 2.- E160A23; 3.- E160A33. $\alpha f$, Coeficiente de fusión; $\alpha f$,Coeficiente de depósito; P, Productividad del proceso; $\psi$, Coeficiente de pérdidas; E, Eficiencia de depósito referida al núcleo del electrodo; Ed, Eficiencia del depósito; Et, Eficiencia total del electrodo; M, Promedio; D, Desviación Estándar. 
El valor de la desviación estándar de la distancia promedio entre conchas demuestra la regularidad en la fenomenología involucrada en el proceso de formación del cordón.

\section{8. Criterios sobre los índices de consumo de los electrodos evaluados con $160 \mathrm{~A}$}

El rendimiento de los electrodos revestidos puede evaluarse a través del cálculo de sus índices de consumo (Tabla 5).

La desviación estándar no supera el $1,23 \%$ en ninguno de los parámetros de consumo, lo cual evidencia un desempeño prácticamente inalterable de este tipo de electrodo bajo las condiciones experimentales establecidas.

\subsection{Criterios sobre el comportamiento de los parámetros eléctricos primarios del arco}

Las variaciones de la longitud del arco, se reflejan en el comportamiento del voltaje y la corriente de soldadura. La raíz cuadrática media de los valores de ambos parámetros (Tabla 6) es comúnmente empleada en términos ingenieriles para la medición de señales eléctricas.

Tabla 6. Comportamiento de la raíz cuadrática media (RMS) del voltaje (Vrms) y la corriente (Irms) de soldadura durante los experimentos realizados a $160 \mathrm{~A}$

\begin{tabular}{|c|c|c|}
\hline Experimento & Irms (A) & Vrms (V) \\
\hline E160A22 & 161,7 & 19,78 \\
\hline E160A23 & 160,91 & 19,97 \\
\hline E160A33 & 162,61 & 19,82 \\
\hline Promedio & 161,74 & 19,86 \\
\hline Desviación estándar & 0,85 & 0,1 \\
\hline
\end{tabular}

La RMS ha sido calculada a partir del conjunto de valores de voltaje y corriente obtenido independientemente de cada ensayo, aproximadamente 150000 valores colectados, exactamente a razón de 5000 muestras por segundo y manteniendo las mismas condiciones experimentales. Una diferencia promedio de $0,85 \mathrm{~A}$ y $0,1 \mathrm{~V}$ en los tres experimentos indica que el comportamiento de los parámetros eléctricos no ha diferido en amplitud en más de un $0,52 \%$ en el caso de la corriente y de un $0,50 \%$ en el caso del voltaje.

\section{Análisis y discusión de Resultados}

En régimen de transferencia metálica por cortocircuitos, la duración del cortocircuito es un parámetro importante, pues mantiene una proporcionalidad directa con la magnitud del aporte metálico en cada período de transferencia.

Un análisis estadístico de las distribuciones probabilísticas empíricas de los nueve ensayos realizados, ha permitido ajustar cada una de las distribuciones a un modelo Lognormal por dos métodos diferentes, que se complementan. Se evidencia una alta reproducibilidad y repetitividad de los resultados (Tabla 2), sugiriendo el mantenimiento de los principales mecanismos fenomenológicos desencadenados durante la soldadura con este tipo de electrodo y sus relaciones en las condiciones experimentales dadas. Una adecuada caracterización de las distribuciones permite analizar una tendencia a disminuir el tiempo de cortocircuito promedio y su desviación estándar a medida que se incrementa la corriente en los ensayos (Tabla 2). Este aspecto coincide plenamente con la teoría tradicional [20] permite seleccionar el régimen de $160 \mathrm{~A}$ como el régimen más estable en el aporte metálico, para el electrodo E6013, marca "RUTH", de diámetro $4 \mathrm{~mm}$. De manera coherente, es posible relacionar el régimen de trabajo utilizado con el patrón de la distribución probabilística del parámetro estudiado (Figura 4 y 5).

En la uniformidad en el aporte metálico (Figuras 6 y 7), influyen las características físico-químicas del electrodo, energéticas del proceso y del equipamiento empleado para ejecutar el mismo [21, 22].

El análisis de forma de los cordones producidos en el régimen más estable (Figuras 6 y 7) demuestra que el volumen de metal aportado por unidad de tiempo es aproximadamente invariante en la zona central de los tres cordones, lo cual es un reflejo de la tendencia observada en las curvas probabilísticas de duración del cortocircuito (Figuras 4 y 5) en tales experimentos.

Una mínima variación en la distancia entre frentes de solidificación sucesivos (Figura 7), evidencia que la velocidad de soldadura se mantiene prácticamente constante pues el frente de solidificación se ubica regularmente espaciado (Tabla 4) a medida que la fuente de calor se aleja del volumen de metal solidificado en cada instante de tiempo. Se aprecian condiciones estables en la velocidad de enfriamiento del baño fundido, donde la capa de escoria juega un papel fundamental [21, 23]. Este comportamiento supone un comportamiento químico, geométrico y térmico regular de la escoria. La conjunción de estos aspectos corrobora la estabilidad en el aporte térmico y la transferencia metálica.

Los índices de consumo de los electrodos (Tabla 5), se determinan teniendo en cuenta las cantidades de metal depositado en el cordón, las pérdidas metálicas [24], etc. El análisis estadístico de estos parámetros demuestra una alta estabilidad del proceso, lo cual refuerza las valoraciones anteriores.

El análisis (Tabla 6) del comportamiento general de los parámetros eléctricos (voltaje y corriente de soldadura), aporta una nueva evidencia de que el voltaje y la corriente de soldadura promediadas, según la Raíz Cuadrática Media en los tres experimentos, es prácticamente invariante (Epígrafe 2.9). Por ende, la longitud del arco en los tres experimentos se comporta aproximadamente constante, lo cual evidencia una alta estabilidad en la taza de fusión del electrodo y la longitud de la copa en la punta del mismo para cada valor de corriente evaluado (Epígrafe 2.1). Estos aspectos corroboran la no interferencia del dispositivo de autoalimentación por gravedad sobre las condiciones impuestas por el propio funcionamiento del consumible durante el proceso.

\section{Conclusiones}

1.El empleo del análisis estadístico por diferentes métodos y algoritmos, ha permitido determinar que el comportamiento 
del parámetro "duración del cortocircuito" en la soldadura con corriente alterna, con el electrodo estudiado se ajusta al modelo de una distribución "Lognormal" y, por tanto, asumir la metodología de cálculo de los estadígrafos de dispersión y localización con un nivel de confidencia de un $95 \%$.

2. La conjugación del análisis estadístico del tiempo de duración del cortocircuito, la zona de influencia térmica, la penetración, la distancia promedio entre los frentes de solidificación del cordón y su ancho y altura de refuerzo, junto a la determinación de los parámetros eléctricos primarios del arco y los índices de consumo de los electrodos, en función del diseño experimental utilizado, permiten obtener relaciones entre el comportamiento eléctrico del arco, el proceso de transferencia de masa y la apariencia del cordón depositado, para extraer criterios sobre la estabilidad del proceso y el desempeño del dispositivo de alimentación empleado.

3. El dispositivo de autoalimentación para electrodos revestidos, diseñado y probado, permite la ejecución repetible y reproducible de cordones de soldadura en posición plana, manteniendo la longitud del arco en función de las características físico-químicas del consumible evaluado en distintos regímenes de trabajo.

\section{Referencias Bibliográficas}

[1] Chu, W.H., Automatización SMAW mediante control Fuzzy para controlador PID con ganancia programada. 2003. p. 108.Tesis Doctoral. China.

[2] Ponomarev, V., Arc Welding Process Statistical Analysis. Methodical Approaches, Analysis Conceptions, Experiences. 1ra Ed Rusia, 1997. p. 27-137.

[3] Souza, M.S.et al. Validación de un sistema computarizado para el control de la calidad de electrodos revestidos, 1995. p 87. Tesis Doctoral. Centro de Ciencias Exactas y Tecnología. Universidad Federal de Uberlandia, Brasil.

[4] N. M. R. DE RISSONE, et al. ANSI/AWS A5.1-91

E6013 Rutile electrodes: The Effect of Calcite. Welding

Journal Supplement. p 113-S - 124-S. Julio 2002

[5] J.P.Farias, et al. The effect of wollastonite on operational characteristics of AWS E6013 electrodes. Journal of the Brazilian Society of Mechanical Sciences and Engineering, V $26 \mathrm{~N}_{\mathrm{o}}$ 3. p 17. Julio/Septiembre. 2004.

[6] García Rodríguez, A.G.P., C. R., Criterios sobre algunas Metodologías de Caracterización Operativa de Electrodos

Revestidos en la Soldadura en Modo de Transferencia Metálica por Cortocircuitos. Soldadura \& Inspeçao, Junio 2008.

[7] Glizmanenco.D.L, Soldadura y Corte de los Metales.

Segunda Ed, URSS. 1962, Moscú. (113) 474.

[8] Cary, H.B., Modern Welding Technology third edition.

1996.

[9] Weman, K., Welding Processes handbook. 2003,

Cambridge: Woodhead Publishing Ltd. 189.

[10] Pessoa, E.C.P. Estudo comparativo do Desempenho de Eletrodos Revestidos E6013,E7024 e E7018 em Soldagem Subacuática em Água Doce do ACO A36 Á Profundidades de 50 E 100 Metros. in Congresso Brasileiro do Engenharia do

Fabricacao. 2003. Uberlandia. Brasil.
[11] C.P.Pessoa, E. Study of Porosity Location in Fresh Water Wet Welds. in 22ND International Conference on Offshore Mechanics and Arctic Engineering. 2003. Cancun, Mexico.

[12] ANSI/AWS, Specification for carbon steel electrodes for shielded metal arc welding, Standard 5.1-91, Miami, Fl,. 1991, American National Standards Institute/American Welding Society.

[13] Advantech, PCLD-8710 User's Manual. 1ra ed, ed. E. Advantech. 1998.

[14] Roca, A.S., Nuevo criterio para el análisis de estabilidad del proceso de soldadura GMAW, empleando técnicas de Emisión Acústica e Inteligencia Artificial, in Facultad Ingeniería Mecánica.Departamento de Tecnología de Construcción de Maquinaria. 2006, Universidad de Oriente: Santiago de Cuba. p. 105.

[15] Advantech, PCI-1710 Series. User's Guide. 1ra ed, ed. E.A. Co. 2001.

[16] Normalización, I.n.d.I.y., Anteproyecto de la Norma Cubana sobre las Características de consumo de los electrodos Revestidos. 1985.

[17] F.J.Medina-Maldonado, e.-a., Disfunción endotelial medida en los miembros inferiores de pacientes con arteriopatía periférica. ANGIOLOGIA, 2007. 59(3): p. 237-244.

[18] J.P.Royston, A toolkit for testing for non-normality in complete and censored samples. The statistician, 1993. 42: p. $37-42$.

[19] A.M.Mood, Introducction to the Theory of Statistics. Third Edition ed. 1974: McGraw-Hill. 540-541.

[20] Norrish, J., Advanced Welding Processes. New Manufacturing Processes and Materials Series, ed. D.A. John Wod. 1992, Philadelphia, New York: IOP Publishing LTD. 499. [21] Grong.Ø, Metallurgical modelling of welding. 1994, Cambridge: The Institute of Materials.

[22] J.F.Lancaster, The physics of welding. Second Edition. 1986. p.340.

[23] Ramírez, J., Soldadura por Arco Manual con Electrodos Revestidos. 2003, Universidad Central de Venezuela.Facultad de Ingeniería. Escuela de Ingeniería. p. 15.

[24] 2401-72, T.C.I.T.I., Covered Electrodes. Determination of the Efficiency metal recovery and the deposition coefficient. 1972. 